\title{
Finite number of Kaluza-Klein modes, all with zero masses
}

\author{
Recai Erdem* \\ Department of Physics, İzmir Institute of Technology \\ Gülbahçe Köyü, Urla, İzmir 35430, Turkey
}

(Dated: November 5, 2018)

\begin{abstract}
Kaluza-Klein modes of fermions in a 5-dimensional toy model are considered. The number of Kaluza-Klein modes that survive after integration over extra dimensions is finite in this space. Moreover the extra dimensional piece of the kinetic part of the Lagrangian in this space induces no mass for the higher Kaluza-Klein modes on contrary to the standard lore.
\end{abstract}

\footnotetext{
*Electronic address: recaierdem@iyte.edu.tr
} 
The use of extra dimension(s) is a popular tool in high energy physics $[1,2,3,4,5,6$, $7,8,9,10]$ because it gives a more tidy picture of nature, that ranges from geometrization of all forces of nature in the spirit of general relativity to a better understanding of the cosmological constant problem, hierarchy problem, fermion generations, Yukawa couplings and flavor etc.. The world we live in is apparently 4-dimensional. Hence extra dimensions (if exist) must be hidden at present (relatively low) energies. The standard way to ensure this is to take the extra dimension(s) be compact and tiny (e.g. a tiny circle). Then, by Fourier theorem, a field in the whole space can be expanded in a tower of particles that are identical except their masses and their profile in the extra dimension(s). Such a tower of a particle (or field) is called a Kaluza-Klein (KK) tower of that particle (or field), and it is an infinite series except in some exceptional cases that need complicated boundary conditions to be satisfied $[11,12,13,14]$. Depending on the boundary conditions the KK tower may contain a zero mode (i.e. a mode that does not depend on the extra dimension(s)) or not. A zero mode does not acquire a mass from the extra dimensional piece of the kinetic part of the Lagrangian while all other modes gain masses of order of $\frac{1}{L}$ where $L$ is the size of the extra dimension. Phenomenological considerations require the masses of the higher KK modes be at least in $\mathrm{TeV}$ scale, and usually in the order of Planck mass for standard model particles [15]. So KK modes except the zero mode can not be identified with the usual particles. Therefore a scheme where the number of KK modes is finite and all gain zero masses from the kinetic part of the Lagrangian would be highly desirable. I this study I present a toy model for fermions where the number of observed KK modes is finite at current energies, and all modes are massless as long as the kinetic part of the Lagrangian is considered.

In the vein of a framework proposed for cosmological constant problem [16, 17, 18, 19] I consider the following 5-dimensional metric

$$
\begin{aligned}
d s^{2}= & g_{B C} d x^{B} d x^{C}=\cos k z\left(g_{\bar{B} \bar{C}} d x^{\bar{B}} d x^{\bar{C}}\right) \\
= & \cos k z\left[g_{\bar{\mu} \bar{\nu}}(x) d x^{\bar{\mu}} d x^{\bar{\nu}}-d z^{2}\right] \\
& B, C, \bar{B}, \bar{C}=0,1,2,3,4 \quad, \quad \bar{\mu}, \bar{\nu}=0,1,2,3
\end{aligned}
$$

where the symbol $x$ with no indices stands for the 4-dimensional coordinates $x^{\bar{\mu}}$. I take the extra dimension be compact and its size be $L$, and $k=\frac{2 \pi}{L}$. Although this metric has singularity at $k z=\frac{\pi}{2}$ this singularity does not survive after integration over the extra dimension $z$ (i.e. at the scales larger than the size of the extra dimension). Moreover the 
location of the singularity at the sharp value, $k z=\frac{\pi}{2}$ suggests that this singularity may be removed by the metric fluctuations in quantum gravity [20]. So given the toy model nature of this study I will not dwell on this technical point further for the sake of a relatively simple framework to study.

I take $g_{\bar{B} \bar{C}}=\eta_{\bar{B} \bar{C}}=\operatorname{diag}(1,-1,-1,-1,-1)$ (i.e. $\left.g_{\bar{\mu} \bar{\nu}}=\eta_{\bar{\mu} \bar{\nu}}\right)$ to have a simple model where one can focus on the essential points of the model. The action for (free) fermionic fields for this space is

$$
\begin{aligned}
S_{f}= & \int(\cos k z)^{\frac{5}{2}} \mathcal{L}_{f} d^{4} x d z \\
= & \int(\cos k z)^{2} i \bar{\chi} \gamma^{a}\left(\partial_{a}+\frac{k}{8} \tan k z\left[\gamma_{4}, \gamma_{a}\right]\right) \chi d^{4} x d z+H . C . \\
& \left\{\gamma^{a}, \gamma^{b}\right\}=2 \eta^{a b}, \quad\left(\eta^{a b}\right)=\operatorname{diag}(1,-1,-1,-1,-1)
\end{aligned}
$$

where H.C. stands for Hermitian conjugate, and the second term is spin connection term (See Appendix A). The small Latin indices $a, b$, etc. refer to the tangent space while the capital Latin indices $A, B$, etc. refer to the space defined by (1). The tangent space in this case coincides with $g_{\bar{B} \bar{C}} d x^{\bar{B}} d x^{\bar{C}}=\eta_{\bar{B} \bar{C}} d x^{\bar{B}} d x^{\bar{C}}$. So the indices with a bar on it also refer to the tangent space in this paper. The action is required to be invariant under the 5-dimensional space-time reflections, namely,

$$
x^{a} \rightarrow-x^{a} \quad, \quad a=0,1,2,3,4
$$

where all coordinates are space-time reflected simultaneously.

$\chi$ may be Fourier decomposed in the extra dimension as

$$
\begin{aligned}
\chi= & \chi_{\mathcal{A}}+\chi_{\mathcal{S}} \\
\chi_{\mathcal{A}}(x, z)= & \sum_{n=-\infty}^{\infty} \chi_{n}^{\mathcal{A}}(x) \sin \left(\frac{1}{2} n k z\right)=\sum_{|n|=1}^{\infty} \tilde{\chi}_{|n|}^{\mathcal{A}}(x) \sin \left(\frac{1}{2}|n| k z\right) \\
\chi_{\mathcal{S}}(x, z)= & \sum_{n=-\infty}^{\infty} \chi_{n}^{\mathcal{S}}(x) \cos \left(\frac{1}{2} n k z\right)=\chi_{0}(x)+\sum_{|n|=1}^{\infty} \tilde{\chi}_{|n|}^{\mathcal{S}}(x) \cos \left(\frac{1}{2}|n| k z\right) \\
& \tilde{\chi}_{|n|}^{\mathcal{A}}(x)=\chi_{n}^{\mathcal{A}}(x)-\chi_{-n}^{\mathcal{A}}(x) \quad, \quad \tilde{\chi}_{|n|}^{\mathcal{S}}(x)=\chi_{n}^{\mathcal{S}}(x)+\chi_{-n}^{\mathcal{S}}(x)
\end{aligned}
$$

(where the absolute value signs in $|n|$ is used to emphasize the positiveness of $n$ in those terms, and half-fractional values in the sum correspond to anti-periodic boundary conditions). The form of $\chi_{n}^{\mathcal{A}(\mathcal{S})}$ is determined by the requirement of covariance under (the spinor 
representation of $S O(3,1)$ and is given by

$$
\begin{aligned}
\chi_{n}^{\mathcal{A}(\mathcal{S})}= & \chi_{0 n}^{\mathcal{A}(\mathcal{S})}+\sum \Gamma^{4} \chi_{4 n}^{\mathcal{A}(\mathcal{S})} \\
& \left\{\Gamma^{B}, \Gamma^{C}\right\}=\frac{2}{\cos k z} \eta^{B C} \quad B, C=0,1,2,3,4
\end{aligned}
$$

where $\Gamma^{B(C)}$ 's are the gamma matrices of $(1)$. However we take $\chi_{n}^{\mathcal{A}(\mathcal{S})}$ simply be $\chi_{0 n}^{\mathcal{A}(\mathcal{S})}$ for the sake of simplicity and it does not essentially change the result as we shall mention when we discuss the masses of the KK modes. Let us return to the main subject after this remark. I take $\chi_{n}^{\mathcal{A}(\mathcal{S})}$ to transform under $(3)$ as

$$
\chi_{n}(x) \rightarrow(-1)^{\lambda_{n}} \mathcal{C} \mathcal{P} \mathcal{T} \chi_{n}(-x) \quad, \quad \lambda_{n}=\frac{1}{2}(-1)^{\frac{n}{2}}
$$

where the upper indices $\mathcal{A}$ and $\mathcal{S}$ are suppressed, and $\mathcal{C P} \mathcal{T}$ denotes the usual 4-dimensional CPT operator (acting on the spinor part of the field). Only the positions of fields (i.e. $x^{a}$ 's) are multiplied by -1 while the orientation of the fields in the space-time remain essentially the same i.e. the spinor part of $\chi$ remains essentially the same. In this respect (3) is the analog of CPT transformation rather than PT transformation in 4-dimensions. The invariance of (2) under (3) requires $i \bar{\chi} \gamma^{a} \partial_{a} \chi$ in $\mathcal{L}_{f}$ be invariant under (3). $i \bar{\chi} \gamma^{\mu} \partial_{\mu} \chi$ is invariant under 4-dimensional CPT. These together imply that $i \bar{\chi} \gamma^{\mu} \partial_{\mu} \chi$ (i.e. $\left.i \bar{\chi} \gamma^{\mu} \chi\right)$ is even under the extra dimensional part of (3). So the possible form of $\mathcal{L}_{f}$ (after requiring it be odd under (3)) is

$$
i \bar{\chi}_{\mathcal{S}} \gamma^{a} \partial_{a} \chi_{\mathcal{S}} \quad, \text { and/or } \quad i \bar{\chi}_{\mathcal{A}} \gamma^{a} \partial_{a} \chi_{\mathcal{A}}
$$

In other words (3) requires $\mathcal{L}_{f}$ be either of the terms in (9) or their linear combination.

Further the invariance of the action under an extra dimensional reflection similar to the one given in $[16,17]$

$$
k z \rightarrow \pi+k z
$$

is imposed. Under (10) the volume element in (2) transforms as

$$
(\cos k z)^{\frac{5}{2}} d^{4} x d z \rightarrow \sqrt{-1}(\cos k z)^{\frac{5}{2}} d^{4} x d z
$$

Then invariance of (2) under (10) requires $i \bar{\chi} \gamma^{a} \partial_{a} \chi$ be even under the same transformation. I impose $\chi$ satisfy anti-periodic boundary conditions [21] i.e. $\chi(z=0)=-\chi(z=L)$. This sets $n$ in $(5,6)$ be odd. Then, the invariance of action (including the quantum paths) under 
(3), (8) and (10) requires the 4-dimensional part of $S_{f}$ be (see Appendix B)

$$
\begin{aligned}
& \sum_{r, s=0}^{\infty} \int d^{4} x i \bar{\chi}_{(2|r|+1)} \gamma^{\bar{\mu}} \partial_{\bar{\mu}} \chi_{(2|s|+1)} \\
& \times 2 \int d z(\cos k z)^{2}\left[\cos \frac{2|r|+1}{2} k z \cos \frac{2|s|+1}{2} k z-\sin \frac{2|r|+1}{2} k z \sin \frac{2|s|+1}{2} k z\right]+H . C . \\
= & \sum_{r, s=0}^{\infty} \int d^{4} x i \bar{\chi}_{(2|r|+1)} \gamma^{\bar{\mu}} \partial_{\bar{\mu}} \chi_{(2|s|+1)} \int_{0}^{L} d z(\cos 2 k z+1) \cos (|r|+|s|+1) k z+H . C . \\
= & \frac{1}{2} \sum_{r, s=0}^{\infty} \int d^{4} x i \bar{\chi}_{(2|r|+1)} \gamma^{\bar{\mu}} \partial_{\bar{\mu}} \chi_{(2|s|+1)} \int_{0}^{L} d z[\cos (|r|+|s|-1) k z]+H . C .
\end{aligned}
$$

where $2 r+1=4 l+1,2 s+1=4 p+3(1, \mathrm{p}=0,1,2, \ldots$.$) or vice versa. Because of the periodicity$ of cosine function the terms in (12) give non-zero contributions after integration over $z$ only if the arguments of cosines are zero. This is possible only when

$$
|r|+|s|-1=0 \quad \Rightarrow \quad r=0 \quad, \quad s=1 \quad \text { or } \quad s=1 \quad, \quad r=0
$$

The result of $z$ integration in (12) is

$$
\frac{L}{2} \int d^{4} x\left[i \bar{\chi}_{1} \gamma^{\bar{\mu}} \partial_{\bar{\mu}} \chi_{3}+i \bar{\chi}_{3} \gamma^{\bar{\mu}} \partial_{\bar{\mu}} \chi_{1}\right]+\text { H.C. }
$$

The diagonalization of (14) results in

$$
\begin{aligned}
& \frac{1}{2} L \int d^{4} x\left[i \bar{\psi} \gamma^{\bar{\mu}} \partial_{\bar{\mu}} \psi-i \overline{\tilde{\psi}} \gamma^{\bar{\mu}} \partial_{\bar{\mu}} \tilde{\psi}\right]+H . C . \\
& \psi=\frac{1}{\sqrt{2}}\left(\chi_{1}+\chi_{3}\right) \quad, \quad \tilde{\psi}=\frac{1}{\sqrt{2}}\left(\chi_{1}-\chi_{3}\right)
\end{aligned}
$$

Hence there are one usual fermion and one ghost fermion in the spectrum.

The $i \bar{\chi} \gamma^{4} \partial_{4} \chi$ part of $\mathcal{L}_{f}$ reduces to $\sin \frac{|n|-|m|}{2} k z$ type of terms as a result of the action of the derivative operator $\partial_{4}$ in (2) (see Appendix A, part 2). This, in turn, results in odd number of sine terms (in the action) that leads to zero after integration over $z$. The number of modes that survive after integration may be increased by changing the extra dimension dependent conformal factor and/or the dimension of the space. For example if the conformal factor in (1) is changed to $\cos ^{2} k z$ then the condition (13) is changed into $r+s-3=0$. The kinetic term induces no mass term in this case as well because the extra dimensional derivatives induce odd number of sine terms in this case as well. The same second term in (2), that is, the spin connection term also induce no mass term because it contains odd number of sine terms as well (see Appendix A, part 1). A similar conclusion should be 
expected for more complicated conformal terms or higher dimensional spaces. In other words no mass is induced for Kaluza-Klein modes through the extra dimensional part of the kinetic term in this model, and similar results are expected for more complicated situations with similar conformal terms and symmetries. Here I have taken $\chi_{n}$ 's be simply given by the first terms in (7). However taking the general form does not change the conclusion because vanishing of the extra dimensional kinetic term after integration follows directly from the extra dimensional coordinates rather than the extra dimensional form of the spinor.

I have introduced an extra dimensional model where only two modes of Kaluza-Klein tower appear at low energies. These modes correspond to a fermion and a ghost fermion. These fermions are massless provided we do not introduce a bulk mass term explicitly. The ghost fermion may be identified by a Lee-Wick [23] or Pauli-Villars [22] type regularization field. These results are quite non-standard both in the emergence of a finite number of Kaluza-Klein modes and the modes higher than zero mode gaining no masses through the extra dimensional piece of kinetic term at low energies where the extra dimensions become directly unobservable. In fact this is also the basic tool to distinguish this scheme from the usual Kaluza-Klein prescription. If nature behaves in the way described here then all Kaluza-Klein modes of a fermion will be observed at short distances smaller than the size of the corresponding extra dimension while only a finite number of these modes will be detected at larger scales after they are produced (even when they are stable or long living so that they can travel large distances before decay). Moreover the coupling of fermions to other particles would vary nonlinearly with distance at the scales smaller than the size of the extra dimension since the screening effect of the conformal factor $\cos k z$ changes nonlinearly at distances below the size of the extra dimension. This would be another characteristic of this type of models. In fact one may easily find different metrics of different form and in different dimensions with finite number of Kaluza-Klein modes (obtained after integration over extra dimensions) and all with zero masses. The aim of this study is to show the possibility of obtaining finite number of Kaluza-Klein modes at low energies, and the possibility of massless Kaluza-Klein modes higher than zero mode. So a relatively simple model where these properties can be observed is studied here rather than a detailed model that is in agreement with phenomenology. I hope different variations of such models with more realistic spectra may be found in future. 


\section{Acknowledgments}

This work was supported in part by Scientific and Technical Research Council of Turkey under grant no. 107T235.

\section{APPENDIX A: POSSIBLE CONTRIBUTIONS TO MASSES DUE TO THE SPIN CONNECTION AND THE EXTRA DIMENSIONAL PART OF THE KINETIC TERM}

\section{Contribution Due to Spin Connection}

The vielbeins, $e_{B}^{a}$, corresponding to the metric, $g_{B C}$ in (1), and those corresponding to its inverse $g^{B C}$ are determined from

$$
g_{B C}=\eta_{a b} e_{B}^{a} e_{C}^{b} \quad, \quad g^{B C}=\eta^{a b} e_{a}^{B} e_{b}^{C}
$$

where the lower indices $a, b$ stand for the tangent space of the original space (e.g. the one defined by (1)). The vielbeins corresponding to the metric in (1) are found to be

$$
e_{B}^{a}=\sqrt{\cos k z} \delta_{B}^{a} \quad e_{a}^{B}=\frac{1}{\sqrt{\cos k z}} \delta_{a}^{B}
$$

where $\delta_{B}^{a}, \delta_{a}^{B}$ are the Kronecker delta, and $\left(\eta_{A B}\right)=\operatorname{diag}(1,-1,-1,-1,-1)$. In curved spaces the derivative term $\partial_{B}$ when acting on spinors is replaced by $D_{B}[24]$

$$
\begin{aligned}
D_{B}= & \partial_{B}+\frac{i}{2} J_{b c} \omega_{B}^{b c} \\
& \text { where } J_{b c}=-\frac{i}{4}\left[\gamma_{b}, \gamma_{c}\right]
\end{aligned}
$$

Here $\gamma_{b(c)}$ are the (flat) tangent space Dirac gamma matrices that are related to the gamma matrices of the original space $\Gamma_{B}$ by

$$
\begin{gathered}
\Gamma_{B}=e_{B}^{a} \gamma_{a} \quad, \quad\left\{\Gamma_{B}, \Gamma_{C}\right\}=2 g_{A B}=\frac{2}{\cos k z} \eta_{B C} \quad, \quad\left\{\gamma_{a}, \gamma_{b}\right\}=2 \eta_{a b} \\
B, C=0,1,2,3,4 \quad, \quad a, b=\overline{0}, \overline{1}, \overline{2}, \overline{3}, \overline{4}
\end{gathered}
$$

where the bars over the integers are used to emphasize that they belong to the tangent space, and $\omega_{B}^{b c}$ 's are the spin connections, that are given by

$$
\omega_{B}^{b c}=\left[e_{K}^{b}\left(\frac{\partial e_{P}^{c}}{\partial x^{B}}\right)-\Gamma_{P B}^{F} e_{K}^{b} e_{F}^{c}\right] g^{K P}
$$


where $\Gamma_{P B}^{F}=\frac{1}{2} g^{F G}\left(g_{P G, B}+g_{B G, P}-g_{P B, G}\right)$ denotes Christoffel symbols, and the commas denote the usual derivative with respect to that coordinate. The non-vanishing $\Gamma_{P B}^{F}$ 's in the space defined by Eq.(1) are

$$
\Gamma_{\nu 4}^{\mu}=-\frac{k}{2} \delta_{\nu}^{\mu} \tan k z, \quad \Gamma_{\mu \nu}^{4}=\frac{k}{2} \eta_{\mu \nu} \tan k z, \quad \Gamma_{44}^{4}=\frac{k}{2} \tan k z
$$

So the spin connection that gives a non-zero contribution is found to be

$$
\omega_{\mu}^{b c}=\frac{k}{2} \tan k z\left[\delta_{4}^{b} \delta_{\mu}^{c}-\delta_{\mu}^{b} \delta_{4}^{c}\right]
$$

The $\omega_{4}^{b c}$ element of spin connection is found to be zero. Then

$$
\begin{aligned}
D_{\mu} & =\partial_{\mu}+\frac{i}{2} J_{b c} \omega_{\mu}^{b c}=\partial_{\mu}+\frac{k}{8} \tan k z\left[\gamma_{\overline{4}}, \gamma_{\bar{\mu}}\right] \quad, \quad D_{4}=\partial_{4} \\
\Gamma^{B} D_{B} & =e_{a}^{\mu} \gamma^{a} D_{\mu}+e_{a}^{4} \gamma^{a} \partial_{4}=\frac{1}{\sqrt{\cos k z}}\left[\gamma^{\bar{\mu}} D_{\bar{\mu}}+\gamma^{\overline{4}} \partial_{\overline{4}}\right]
\end{aligned}
$$

where the upper case indices B,C,F etc. denote the space-time coordinates while the lower case indices a,b,c etc. and the indices with a bar over e.g. $\bar{B}, \overline{4}$ etc. denote the tangent space. Although there is a bar over 4 in (A7) that bar is omitted in (2) to simplify the notation. Therefore the result may be written a more compact form as in Eq.(2) where $\omega_{4}^{a b}$ gives null contribution.

After using Eqs. (A7) and (A8 one obtains Eq.(2). It is evident from (2) and Eq.(A6) that the integration of the spin connection term $e_{a}^{\mu} \gamma^{a} \omega_{\mu}^{b c} J_{b c}$ over the extra dimension $z$ is proportional to

$$
\int_{0}^{2 \pi}(\cos k z)^{2} \tan k z d(k z)=0
$$

In other words the spin connection term dose not contribute to the masses of $\psi, \tilde{\psi}$ of Eq.(16) at (relatively low energies) where the extra dimension can not be seen.

\section{Contribution Due to the Extra Dimensional Part of the Kinetic Term}

The extra dimensional part of the kinetic term for the action of the field $\chi$ is

$$
\begin{aligned}
& \int(\cos k z)^{\frac{5}{2}} i \bar{\chi} \gamma^{4} \partial_{4} \chi d^{4} x d z=\sum_{r, s=0}^{\infty} \int d^{4} x i \bar{\chi}_{(2|r|+1)} \gamma^{4} \chi_{(2|s|+1)} \int d z(\cos k z)^{2} \\
& \times\left\{\left(\cos \frac{2|r|+1}{2} k z+\sin \frac{2|r|+1}{2} k z\right) \partial_{4}\left(\cos \frac{2|s|+1}{2} k z-\sin \frac{2|s|+1}{2} k z\right)\right. \\
+ & \left.\left(\cos \frac{2|r|+1}{2} k z-\sin \frac{2|r|+1}{2} k z\right) \partial_{4}\left(\cos \frac{2|s|+1}{2} k z+\sin \frac{2|s|+1}{2} k z\right)\right\}
\end{aligned}
$$




$$
\begin{aligned}
= & -k \sum_{r, s=0}^{\infty}(2|s|+1) \int d^{4} x i \bar{\chi}_{(2|r|+1)} \gamma^{4} \chi_{(2|s|+1)} \int d z(\cos k z)^{2} \\
& {\left[\cos \frac{2|r|+1}{2} k z \sin \frac{2|s|+1}{2} k z+\sin \frac{2|r|+1}{2} k z \cos \frac{2|s|+1}{2} k z\right]=0 }
\end{aligned}
$$

where the H.C. symbol (in Eq.(12)) for the addition the Hermitian conjugate of (B4) to

itself is suppressed. So the extra dimensional piece of the kinetic term in this paper does not contribute to the masses of $\psi$ or $\tilde{\psi}$ at length scales larger than the size of the extra dimension.

\section{APPENDIX B: DERIVATION OF EQ.(12)}

It is observed that

$$
\begin{aligned}
\text { as } \quad k z \rightarrow \pi+k z & \\
\text { i) if } n=4 l+1 \Rightarrow & \left(\cos \frac{n}{2} k z+\sin \frac{n}{2} k z\right) \rightarrow\left(\cos \frac{n}{2} k z-\sin \frac{n}{2} k z\right) \\
& \left(\cos \frac{n}{2} k z-\sin \frac{n}{2} k z\right) \rightarrow-\left(\cos \frac{n}{2} k z+\sin \frac{n}{2} k z\right) \\
\text { ii }) \text { if } n=4 l+3 \Rightarrow & \left(\cos \frac{n}{2} k z+\sin \frac{n}{2} k z\right) \rightarrow-\left(\cos \frac{n}{2} k z-\sin \frac{n}{2} k z\right) \\
& \left(\cos \frac{n}{2} k z-\sin \frac{n}{2} k z\right) \rightarrow\left(\cos \frac{n}{2} k z+\sin \frac{n}{2} k z\right) \\
& l=0,1,2, \ldots . .
\end{aligned}
$$

The requirement that the action (2) be invariant under (8)requires $n=4 l+1$ type of modes couple to $m=4 p+3$ type of modes. In the light of this observation the combination that is invariant under $(10)$ is

$$
\begin{aligned}
& \left\{\left(\cos \frac{2|r|+1}{2} k z+\sin \frac{2|r|+1}{2} k z\right)\left(\cos \frac{2|r|+1}{2} k z-\sin \frac{2|r|+1}{2} k z\right)\right. \\
+ & \left.\left(\cos \frac{2|r|+1}{2} k z-\sin \frac{2|r|+1}{2} k z\right)\left(\cos \frac{2|s|+1}{2} k z+\sin \frac{2|s|+1}{2} k z\right)\right\} \\
= & 2\left[\cos \frac{2|r|+1}{2} k z \cos \frac{2|s|+1}{2} k z-\sin \frac{2|r|+1}{2} k z \sin \frac{2|s|+1}{2} k z\right]
\end{aligned}
$$

where

$$
\begin{aligned}
& 2|r|+1=4 l+1 \text { and } 2|s|+1=4 p+3 \\
& \text { or } 2|r|+1=4 l+3 \text { and } 2|s|+1=4 p+1 \\
& l, p=0,1,2,3, \ldots \ldots .
\end{aligned}
$$


So the 4-dimensional part of $S_{f}$ becomes

$$
\begin{aligned}
& \sum_{r, s=0}^{\infty} \int d^{4} x i \bar{\chi}_{(2|r|+1)} \gamma^{\bar{\mu}} \partial_{\bar{\mu}} \chi_{(2|s|+1)} \int d z(\cos k z)^{2} \\
& \times\left\{\left(\cos \frac{2|r|+1}{2} k z+\sin \frac{2|r|+1}{2} k z\right)\left(\cos \frac{2|r|+1}{2} k z-\sin \frac{2|r|+1}{2} k z\right)\right. \\
+ & \left.\left(\cos \frac{2|r|+1}{2} k z-\sin \frac{2|r|+1}{2} k z\right)\left(\cos \frac{2|s|+1}{2} k z+\sin \frac{2|s|+1}{2} k z\right)\right\}
\end{aligned}
$$

where the H.C. symbol (as in Eq.(A10)) is suppressed. (B4) after use of (B2) results in (12). It is evident from (12) that the resulting Lagrangian has the form required by (9) as well.

[1] T. Kaluza, On the problem of unity in physics Sitzungsber. Preuss. Akad. Wiss. Berlin (Math. Phys.) K41, 966 (1921)

[2] O. Klein, Quantum theory and five-dimensional theory of Relativity, Z. Phys. 37, 895 (1926)

[3] M.J. Duff, Kaluza-Klein theory in perspective, in Stockholm 1994, The Oskar Klein centenary pp 22-35, preprint Arxiv hep-th/9410046

[4] N. Arkani-Hamed, S. Dimopoulos, G. Dvali, The hierarchy problem and new dimensions at a millimeter, Phys. Lett. B 429, 263 (1998), preprint ArXiv hep-ph/9803315

[5] I. Antoniadis, N. Arkani-Hamed, S. Dimopoulos, G. Dvali, New dimensions at a millimeter to a Fermi and superstrings at a TeV, Phys. Lett. B 436, 257 (1998), preprint ArXiv hep$\mathrm{ph} / 9804398$

[6] N. Arkani-Hamed, S. Dimopoulos, G. Dvali, Phenomenology, astrophysics and cosmology of theories with submillimeter dimensions and TeV scale quantum gravity, Phys. Rev. D 59, 086004 (1999), preprint ArXiv hep-ph/9807344

[7] L. Randall, R. Sundrum, A Large mass hierarchy from a small extra dimension, Phys. Rev. Lett. 83, 3370 (1999)

[8] L. Randall, R. Sundrum, An Alternative to compactification; Phys. Rev. Lett. 83, 4690 (1999)

[9] Y. Uehara, A mini review of constraints on extra dimensions, Mod. Phys. Lett. A 17, 1551 (2002)

[10] T. Flacke, D. Hooper and J. Marc-Russell, Improved bounds on universal extra dimensions and consequences for LKP dark matter, Phys. Rev. D 73, 095002 (2006), Erratum-ibid. D74, $019902(2006)$ 
[11] F. Grard, J. Nuyts, Elementary Kaluza-Klein towers revisited, Phys. Rev. D 74, 124013 (2006)

[12] F. Grard, J. Nuyts, Warped Kaluza-Klein towers revisited, Phys. Rev. D 76, 124022 (2007)

[13] F. Grard, J. Nuyts, Kaluza-Klein towers in warped spaces with singularities, arXiv:0712.3692

[14] F. Grard, J. Nuyts, Kaluza-Klein towers for spinors in flat space, arXiv:0803.1741

[15] I. Antoniadis, A possible new dimension at a few TeV, Phys. Lett. B 246, 377 (1990)

[16] R. Erdem, A symmetry for vanishing cosmological constant in an extra dimensional toy model, Phys. Lett. B 621, 11 (2005), hep-th/0410063

[17] R. Erdem, A symmetry for vanishing cosmological constant: Another realization, Phys. Lett. B 639, 348 (2006), gr-qc/0603080

[18] R. Erdem, A symmetry for vanishing cosmological constant, J. Phys. A 40, 6945 (2007), gr-qc/0611111

[19] R. Erdem, A way to get rid of cosmological constant and zero-point energy problems of quantum fields through metric reversal symmetry, J. Phys. A 41, 235401 (2008), arXiv:0712.2989

[20] E.I. Guendelman and A.B. Kaganovich, Transition to zero cosmological constant and phantom dark energy as solutions involving change of orientation of space-time manifold, Class. Quant. Grav. 25, 235015 (2008), arXiv:0804.1278

[21] P. Di Francesco, P. Mathieu, D. Sen echal, Conformal Field Theory, pp. 168-171 (Springer, New York, 1997)

[22] W. Pauli and F. Villars, On the Invariant Regularization in Quantum Field Theory, Rev. Mod. Phys. 21, 434 (1949)

[23] T.D. Lee and G.C. Wick, Finite Theory of Quantum Electrodynamics, Phys. Rev. D 2, 1033 (1970)

[24] This may be found in many textbooks although the conventions may vary a little. Our convention is that of

S. Weinberg Quantum Theory of Fields Vol.3, (Cambridge Univ. Press, New York, 2000) 